\title{
Four-branch Star Neutral Current Hybrid Power Filter and Var Compensator
}

\author{
P. Rodriguez ${ }^{1}$ \\ Member IEEE \\ R. Teodorescu ${ }^{2}$ \\ Senior Member IEEE
}
(1) Technical University of Catalonia
C/ Colom 1 08222, Terrassa, Spain
prodriguez@ee.upc.edu

\author{
J. I. Candela ${ }^{1}$ \\ Member IEEE \\ F. Blaabjerg
Fellow IEEE
}

\author{
A. Luna ${ }^{1}$ \\ Student Member IEEE \\ J. Rocabert ${ }^{1}$ \\ Student Member IEEE
}

(2) Aalborg University

Pontoppidanstraede 101, 9220 Aalborg, Denmark ret@iet.aau.dk

\begin{abstract}
This paper presents a solution for filtering current harmonics in three-phase four-wire networks based on the usage of a four-branch star (FBS) filter topology. The specific layout of single-phase inductors and capacitors in this filter topology allows achieving a power filter with two independent and simultaneous resonance frequencies, i.e., one for positive/negative-sequence and another one for zero-sequence components. The FBS filter topology can work either as a passive filter, or as a hybrid filter, when its performance is improved by integrating a power converter into its structure. The paper analyzes the FBS topology and derives fundamental concepts about the control of a generic FBS hybrid power filter. From this analysis, a neutral current hybrid power filter and var compensator is presented as an illustrative example applying the FBS filtering topology to a three-phase four-wire system. An evaluation using simulation and experimental results is conducted as well.
\end{abstract}

Index Terms-- Power system harmonics, power line filters, hybrid power filters, passive power filters, active power filters, reactive power control.

\section{INTRODUCTION}

Current harmonics in electric power networks have become a serious problem in terms of power quality, reliability and continuity of supply. As a consequence, several standard regulating maximum harmonic levels in distribution networks have been proposed in the last decades [1]-[3]. This significant growing of current harmonics mostly results from the widespread usage of nonlinear loads. Discharge lamps and power electronics based equipments are two frequent examples of nonlinear loads in residential, commercial and industrial facilities. Currents harmonics have also a significant effect on medium and low voltage networks due to the existence of distorting singular loads such as furnace ovens or big frequency line rectifiers.

Three-phase three-wire nonlinear loads generate positive/negative-sequence (pn-seq) current harmonics. These harmonics give rise to resonances, voltage distortion, overheating, losses increasing, malfunctions and premature ageing of electrical equipments. Single-phase nonlinear loads are usually connected between one of the phases and the neutral conductors and, in addition to the pn-seq harmonics, they also originate zero-sequence (z-seq) current harmonics typically $3^{\text {rd }}-, 9^{\text {th }}$ - and $15^{\text {th }}$-harmonics. Harmonic currents of orders multiple of three resulting from several single-phase loads are summed up in the neutral conductor, which can result in a harmonic current in the neutral conductor up to three times higher than in the phase ones. Z-seq current harmonics, as well as causing typical problems related to pnseq harmonics, can also give rise to overload of the neutral conductor, common-mode neutral to earth voltages, increasing of phase voltage distortion and transformers overheating [4].

Some of the techniques commonly applied to neutral current harmonics cancellation in three-phase four-wire electrical networks are: shunt zig-zag reactors [5], passive LC power filters with a very high quality factor [6], $3^{\text {rd }}$ harmonic blocking power filters in series with the neutral conductor [7] and active and hybrid filters [8]-[10].

This paper presents a technique for filtering current harmonics in three-phase four-wire networks based on the usage of a four-branch star (FBS) filter topology. The FBS topology is characterized by a particular layout of singlephase inductors and capacitors which allows achieving a power filter with two independent resonance frequencies, i.e., one for pn-seq and another one for z-seq components, but without using any specific power transformer or special electromagnetic device. Resonant LC cells in this power filter simultaneously drain away current harmonics with at least two different frequencies and sequences. Inductors and capacitors are designed to drain off $5^{\text {th }}$ and/or $7^{\text {th }}$ and/or $11^{\text {th }}$ and/or $13^{\text {th }}$ order harmonics with pn-seq and $3^{\text {rd }}$ and $/$ or $9^{\text {th }}$ and/or $15^{\text {th }}$ order harmonics with $z$-seq. The FBS filter topology is suitable for working either as a passive filter, when only passive components are employed, or as a hybrid filter, when its behaviour is improved by integrating a power converter into its structure. In the following, the FBS topology is analyzed and a neutral current hybrid power filter is presented as an illustrative example applying the FBS filtering topology to a three-phase four-wire system. 


\section{II. THE FOUR-BRANCH STAR POWER FILTER TOPOLOGY}

\section{A. General structure of the FBS power filter}

The generic structure of the shunt power filter topology proposed in this paper is shown in Fig. 1. The power filter presents a four-branch star (FBS) topology consisting of three phase-branches and one neutral-branch. Three identical single-phase impedances $Z_{f}$ are connected in the phasebranches whereas the fourth single-phase impedance $Z_{n}$ is connected in the neutral-branch. In Fig. 1, the FBS power filter is connected to a generic three-phase network in which pn-seq voltage components, $\mathbf{u}_{12}=\left[u_{a o}, u_{b o}, u_{c o}\right]$, and z-seq voltage components, $u_{0}$, have been represented separately for simplifying the analysis presented in the following.

When only pn-seq components are considered in the circuit of Fig. 1, i.e. with $u_{0}=0$, the center nodes at the source and filter sides $\left(o-o^{\prime}\right)$ are virtually connected and hence $v_{o o},=0$. Therefore, the pn-seq impedance of the FBS power filter $\vec{Z}_{12}$ is given by:

$$
\vec{Z}_{12}=\frac{\vec{U}_{12}}{\vec{I}_{12}}=\frac{\vec{U}_{f o}}{\vec{I}_{f}}=\frac{\vec{U}_{f o^{\prime}}}{\vec{I}_{f}}=\vec{Z}_{f} \text { with } f=\{a, b, c\},
$$

being $\vec{U}_{12}$ and $\vec{I}_{12}$ the pn-seq voltage and current phasors affecting to the FBS power filter, respectively. Likewise, when only the z-seq component is considered in the circuit, namely, when $\mathbf{u}_{12}=0$, the z-seq impedance of the FBS power filter, $\vec{Z}_{0}$, at a certain frequency is given by:

$$
\vec{Z}_{0}=\frac{\vec{U}_{0}}{\vec{I}_{0}}=3 \frac{\vec{U}_{o n}}{\vec{I}_{n}}=\frac{\vec{U}_{o^{\prime} n}}{\vec{I}_{n}} \frac{\vec{Z}_{f}+3 \vec{Z}_{n}}{\vec{Z}_{n}}=\vec{Z}_{f}+3 \vec{Z}_{n}
$$

where $\vec{U}_{0}$ and $\vec{I}_{0}$ are the z-seq voltage and current phasors affecting to the FBS filter, respectively.

Single-phase impedances constituting the FBS power filter could consist in complex LC resonant cells with multiple resonance frequencies. Connection of resonant cells according to FBS topology will give rise to two types of resonance frequencies, i.e., one type of resonance frequencies for the pn-seq components and another one for the z-seq components. Therefore, the shunt passive power filter with FBS topology is able to perform selective filtering of current harmonics by means of setting up low impedance paths for specific current components with specific frequencies and sequences.

Even though the resonant cells composing the FBS filter branches can be really complex in certain applications, a reasonably good filtering characteristic is obtained in practice when such resonant cells present a single resonance frequency. In this presentation of the FBS power filter, simple resonant cells will be considered for the sake of simplifying further explanations. A regular implementation of the FBS power filter based on simple series LC resonant cells is presented in Fig. 2. In this case, phase and neutral

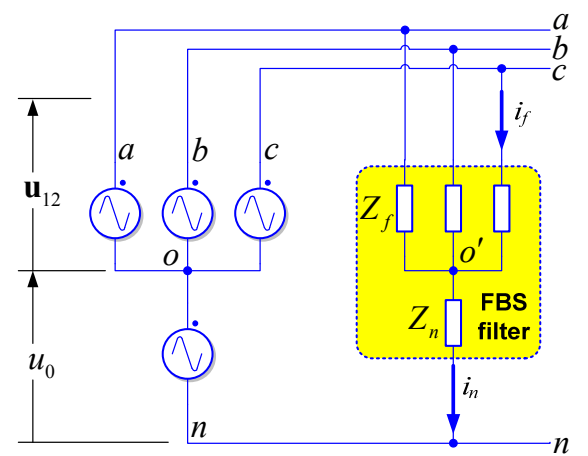

Fig. 1. FBS power filter with generic branch impedances

impedances, $Z_{f}$ and $Z_{n}$ respectively, are given by:

$$
\vec{Z}_{f}=R_{f}+j\left(L_{f} \omega-\frac{1}{C_{f} \omega}\right), \vec{Z}_{n}=R_{n}+j\left(L_{n} \omega-\frac{1}{C_{n} \omega}\right) .
$$

Pn-seq and z-seq impedances of the FBS network can be calculated by substituting impedances of (3) into (1) and (2), this is:

$$
\begin{gathered}
\vec{Z}_{12}=R_{f}+j\left(L_{f} \omega-\frac{1}{C_{f} \omega}\right) \\
\vec{Z}_{0}=\left(R_{f}+3 R_{n}\right)+j\left[\left(L_{f}+3 L_{n}\right) \omega-\frac{1}{\omega}\left(\frac{1}{C_{f}}+\frac{3}{C_{n}}\right)\right] .
\end{gathered}
$$

Impedance expressions of (4) and (5) evidence that the FBS filter of Fig.2 presents a resonance frequency for pn-seq components and another one for the z-seq components. These resonance frequencies are:

$$
\begin{gathered}
f_{12}=\frac{1}{2 \pi} \frac{1}{\sqrt{L_{f} C_{f}}}, \\
f_{0}=\frac{1}{2 \pi} \frac{1}{\sqrt{\left(L_{f}+3 L_{n}\right)\left(\frac{C_{f} C_{n}}{C_{n}+3 C_{f}}\right)}}
\end{gathered}
$$

being $f_{12}$ the resonance frequency for pn-seq components, and $f_{0}$ the resonance frequency for z-seq components.

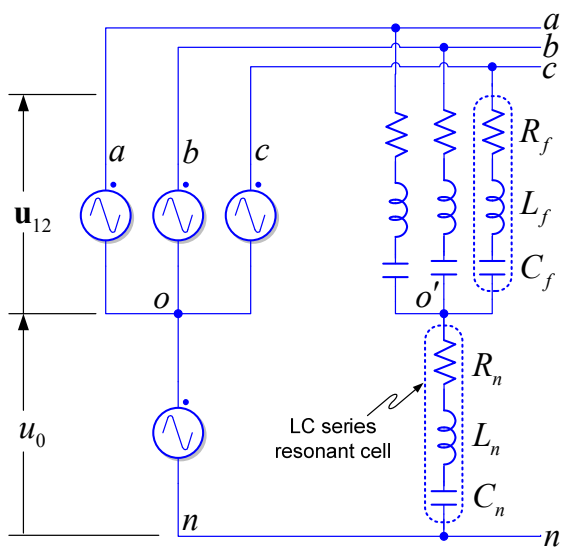

Fig. 2. FBS power filter based on simple series LC resonant cells 


\section{B. Particular implementation of the FBS power filter}

Several variants of power filters can be derived from the generic FBS filter structure of Fig. 2. One of these variants is presented in Fig. 3. This specific configuration of the FBS filter is suitable for both cancellation of z-seq current harmonics at frequency $f_{0}$ and compensation of reactive power at the fundamental grid frequency. In this implementation, the phase-branches are capacitors and an inductor is connected to the neutral-branch. Resistances have been intentionally omitted in Fig. 3 since they are not of interest for calculating the resonance frequency of the $3 C_{f}+L_{n}$ resonant circuit. Z-seq resonance frequency for the FBS power filter of Fig. 3 can be calculated by:

$$
f_{0}=\frac{1}{2 \pi} \frac{1}{\sqrt{3 L_{n} C_{f}}} .
$$

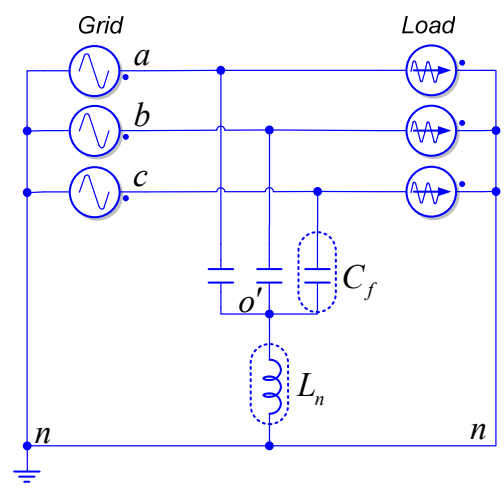

Fig. 3. FBS power filter suitable for $z$-seq current harmonics cancellation and var compensation.

\section{FBS NEUTRAL CURRENT HYBRID POWER FILTER AND VAR COMPENSATOR}

The FBS passive power filter previously presented can offer a fairly good performance in cancelling neutral current harmonics under optimal operating conditions. However, the filtering characteristic of the FBS passive power filter is affected by typical problems of passive filters, i.e., its filtering capability depends on the value of the grid impedance, there is a risk of resonance, retuning is necessary due to ageing and tolerances, etc. A solution to overcome these drawbacks consists in integrating a VSI into the filter structure. This filtering system is known as a hybrid power filter [11]. A properly designed and well controlled VSI can generate any voltage-current relationship at its output, obviously provided that it works inside its operative range. Therefore, such VSI could be understood as a 'virtual impedance' integrated into the original structure of the passive filter. This virtual impedance improves the behaviour of the original passive filter by increasing its capability for draining off current harmonics at frequencies different from the resonance frequencies, compensating drifts in the passive filter parameters, and damping oscillations due to resonance phenomena.

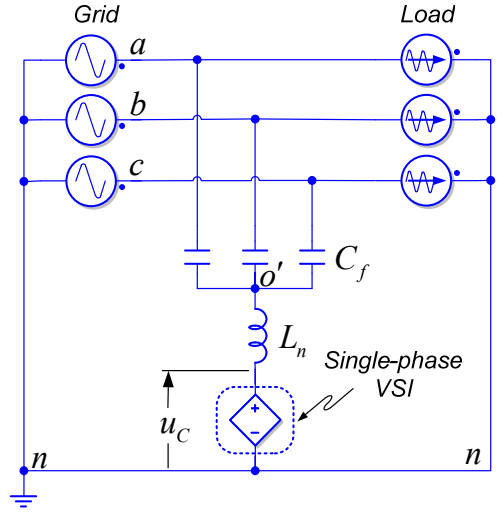

Fig. 4. Neutral current hybrid power filter and var compensator with FBS topology.

Fig. 4 shows a particular implementation of the FBS hybrid power filter suitable for cancellation of current harmonics in the neutral conductor. In this implementation, the single-phase VSI in the neutral-branch of the FBS structure improves the z-seq filter characteristic. Moreover, the three-phase capacitor-bank provides capacitive var compensation to the grid. Detuning inductors can be connected in series with the capacitors of the capacitor-bank to reduce the risk of parallel resonances with the grid. Such inductors are not included in Fig. 4 for simplification.

\section{NeUtRAL CURRENT CONDITIONING WITH THE FBS HYBRID POWER FILTER}

The control algorithm of the filter of Fig. 4 is represented in a general form by means of the diagram of Fig. 5(a), where current harmonics at both the load-side $\left(i_{L}\right)$ and the grid-side $\left(i_{G}\right)$ are considered.

The resonant circuit $3 C_{f}+L_{n}$ of the FBS hybrid power filter of Fig. 5 offers very low impedance at the tuning frequency $f_{0}$ for z-seq currents. Therefore, a low dc-link voltage -only about $10 \%$ of the grid voltage, is necessary in the VSI to inject significant levels of current at the frequency $f_{0}$ into the neutral conductor. However, impedance offered by the resonant circuit grows as frequency goes away from the resonance one. As a result, the high frequency current ripple injected by the FBS hybrid power filter into the grid is very

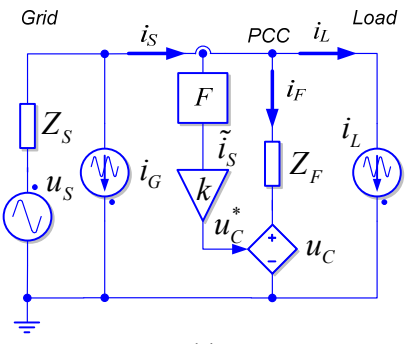

(a)

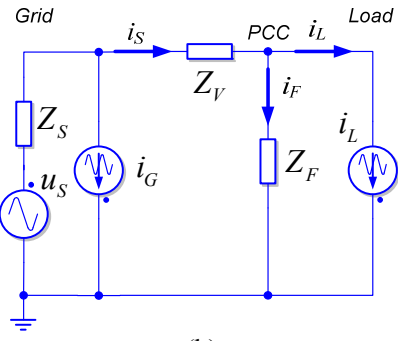

(b)
Fig. 5. (a) Simple control diagram of the FBS hybrid power filter, (b) Equivalent circuit with a virtual impedance $Z_{V}$ resulting from the control law. 
low. However, the FBS hybrid power filter only can compensate a limited range of the current harmonics flowing trough the neutral conductor. This is the reason why a current processing block $(F)$ is necessary to extract those individual frequencies suitable to be filtered $\left(\tilde{i}_{S}\right)$ from the controlled grid current $\left(i_{S}\right)$.

A simple proportional regulator with gain $k$ is used in this work to set the reference voltage $u_{C}^{*}$ of the VSI for injecting a proper compensating current into the neutral conductor. Thus, the control law of the system of Fig. 5(a) is given by:

$$
u_{C}=Z_{V} \cdot i_{S} ; Z_{V}=\mu \cdot k \cdot F,
$$

where $\mu$ is the gain of the VSI.

A straightforward analysis of the diagram shown in Fig. 5(a) conducts to the following transfer function:

$$
i_{S}=\frac{1}{Z_{F}+Z_{S}+Z_{V}}\left(i_{L} Z_{F}-i_{G} Z_{S}+u_{S}\right) \text {, }
$$

where

$$
\begin{gathered}
\vec{Z}_{F}=\vec{Z}_{0}=3 R_{n}+j\left(3 L_{n} \omega-\frac{1}{C_{f} \omega}\right) \text { and } \\
\vec{Z}_{S}=R_{S}+j L_{S} \omega
\end{gathered}
$$

being $R_{S}$ and $L_{S}$ the resistance and inductance presented by the grid, respectively.

Transfer function of (10) reveals that integration of a VSI controlled by the simple control law of (9) into the passive FBS filter structure is equivalent to inserting a virtual impedance $Z_{V}$ upstream of the point of common coupling (PCC) between the hybrid power filter and the grid, as shown in Fig. 5(b). This virtual impedance increases the capability of the hybrid power filter for filtering the load-side current harmonics, isolates the hybrid power filter from the gird-side current harmonics and reduces the risk of resonances between the resonant cells and the grid impedance [12].

Performances of the FBS hybrid power filter presented in this work can be improved by implementing an enhanced controller consisting of individual regulators with a different gain for each of the current harmonics to be compensated [13][14].

\section{PERFORMANCE STUDY OF THE FBS NEUTRAL CURRENT HYBRID POWER FILTER AND VAR COMPENSATOR}

A preferred implementation of the FBS neutral current hybrid power filter and var compensator is shown in Fig. 6. As previously mentioned, this FBS hybrid power filter provides both var compensation at the fundamental grid frequency and current harmonics cancellation in a controlled neutral conductor. To evaluate the performance of the proposed hybrid power filter, injection of current harmonics at both the load- and the grid-side of the neutral conductor is considered in following simulations. Three single-phase diode rectifiers are connected to the grid by the switch SW1 in order to inject current harmonics in the load-side neutral conductor $\left(i_{L n}\right)$. Moreover, another single-phase diode rectifier injects current harmonics in the grid-side neutral conductor $\left(i_{G n}\right)$. As shown in Fig. 6, the controlled neutral conductor is that one connecting the PCC to the grid and thus the FBS hybrid power filter should cancel out harmonics of the neutral current $i_{S n}$. In this particular application, a very simple single-leg VSI is used for injecting the z-seq compensation current into the grid -with the negative rail of its dc-bus connected to the neutral conductor. The z-seq resonance frequency of the FBS resonant circuit $3 C_{f}+L_{n}$ of Fig. 6 is given by (8). The resonance phenomenon allows controlling z-seq current injected into the grid by using a low dc-link voltage level -about $10 \%$ of the line grid voltage. The output voltage of the single-leg VSI of Fig 6, referenced to the negative dc-bus rail, is formed by both ac and dc components. The dc component is blocked by the capacitors $C_{f}$ of the capacitors-bank and it does not inject any dc current into the grid. The ac component of the VSI output voltage has a maximum range of $\pm u_{d c} / 2$ and is in charge of injecting z-seq current into the grid in order to cancel out current harmonics flowing through the controlled neutral conductor. Therefore, the gain of the VSI of Fig. 6 is given by $\mu=u_{d c} / 2$.

The controller used in the FBS power filter subject of study in this work is highlighted in Fig. 6. This controller responds to the control law of (9) when applied to the neutral current. As previously mentioned, the low dc-link voltage of the VSI used in this FBS hybrid power filter makes it not suitable for injecting significant current levels at the fundamental frequency into the grid. Since the neutral current at the fundamental frequency can become notable in unbalanced three-phase four-wire system, a narrow notch filter (NF) tuned at the fundamental frequency was used in the FBS power filter controller of Fig. 6 to eliminate the influence of such fundamental current component in the filter control loop. In this manner, the low dc-link voltage is exclusively employed for efficiently injecting current harmonics at frequencies placed close to resonance frequency of the resonant cell.

The switch SW2 in Fig. 6 allows selecting between the hybrid mode (position 1) and the passive mode (position 0) of the FBS power filter. The reference voltage $u_{C n}^{*}$ is equal zero in the passive mode and hence the VSI does not contribute to the filtering action. Therefore, the virtual impedance $Z_{V}$ in (10) can be considered equal zero in the passive mode. In the hybrid mode, the FBS power filter controller provides the proper reference voltage $u_{C n}^{*}$ to the VSI in order to cancel out harmonics of the neutral current $i_{S n}$. If the reference voltage $u_{C n}^{*}$ was modified in order to additionally regulate the dc-link voltage level, the filtering characteristic of the FBS hybrid power filter would be clearly deteriorated. For this reason, no control loop was added to the FBS power filter controller of Fig. 6 for regulating the dclink voltage level and a simple three-phase diode rectifier 
was used instead to supply the dc-link voltage to the VSI. This dc-link diode rectifier was intentionally not represented in Fig. 6. The dc-link diode rectifier was connected to the grid by means of a small $\Delta y$ transformer. The primary current of this transformer is negligible in respect to the load current since it mainly covers the power losses of the FBS hybrid power filter.

Two different values for the grid impedance were considered in simulation in order to evaluate the effectiveness of the proposed neutral current power filter with FBS topology under different connection conditions. Grid parameters for both study cases are given in Table I.

TABLE I

GRID PARAMETERS IN THE TWO STUDY CASES

\begin{tabular}{ccccc}
\hline \multicolumn{5}{c}{ GRID PARAMETERS IN THE TWO STUDY CASES } \\
Study Case & $\begin{array}{c}\text { Nominal } \\
\text { power }\end{array}$ & $\begin{array}{c}\text { Line } \\
\text { Voltage }\end{array}$ & $\begin{array}{c}\text { Line } \\
\text { resistance } \\
(1.5 \%)\end{array}$ & $\begin{array}{c}\text { Line } \\
\text { inductance } \\
(6.25 \%)\end{array}$ \\
\hline A (weak grid) & $10 \mathrm{~kW}$ & $400 \mathrm{~V} / 50 \mathrm{~Hz}$ & $240 \mathrm{~m} \Omega$ & $3,2 \mathrm{mH}$ \\
B (strong grid) & $100 \mathrm{~kW}$ & $400 \mathrm{~V} / 50 \mathrm{~Hz}$ & $24 \mathrm{~m} \Omega$ & $0,32 \mathrm{mH}$ \\
\hline \hline
\end{tabular}

Whatever the value considered for the grid impedance from Table I, the three single-phase load-side rectifiers of the system of Fig. 6 demand $2.5 \mathrm{~kW}$ and inject an almost sinusoidal current $i_{L n}$ in the neutral conductor at three times the fundamental grid frequency with a rms value of 4.9A. Additionally, the single-phase grid-side rectifier works in discontinuous mode injecting a highly distorted current $i_{G n}$ in the neutral conductor with a rms value of $1 \mathrm{~A}$. Table II shows the main parameters used for simulating the FBS neutral current power filter and var compensator. Applying (8) to this set of parameters, the z-seq resonance frequency $f_{0}$ of the FBS resonant cell is set to $150 \mathrm{~Hz}$. Reactive power supplied by the capacitors-bank is $Q_{F}=1.7 \mathrm{kvar}$ for a $400 \mathrm{~V} / 50 \mathrm{~Hz}$ grid.

TABLE II

PARAMETERS OF THE FBS NEUTRAL CURRENT POWER FILTER

\begin{tabular}{cc}
\hline \multicolumn{2}{c}{ AND VAR COMPENSATOR } \\
\hline Parameter & Value \\
\hline Capacitors-bank capacitance $\left(C_{f}\right)$ & $33.5 \mu \mathrm{F}$ \\
Filter neutral-branch inductance $\left(L_{n}\right)$ & $11.3 \mathrm{mH}$ \\
Filter neutral-branch resistance $\left(R_{n}\right)$ & $320 \mathrm{~m} \Omega$ \\
Rectified dc-link voltage $\left(U_{d c}\right)$ & $45 \mathrm{~V}$ \\
Dc-link capacitor $\left(C_{d c}\right)$ & $10 \mathrm{mF}$ \\
Switching frequency $\left(f_{S W}\right)$ & $14.4 \mathrm{kHz}$ \\
\hline \hline
\end{tabular}

Finally, parameters of the FBS power filter controller are shown in Table III.

From all previous settings, Fig. 7 shows some simulation results for the neutral current power filter and var compensator of Fig. 6. Three different operating modes can be differenced in plots of Fig. 7. In the mode $1(t<0.45 \mathrm{~s})$, the switch SW1 is open and SW2 $=0$, which implies that the
TABLE III

\begin{tabular}{cc} 
PARAMETERS OF THE FBS POWER FILTER CONTROLLER \\
\hline \hline Parameter & Value \\
\hline Proportional gain $(k)$ & 1.0 \\
Notch filter order & 4 \\
Notch filter center frequency $\left(f_{N F}\right)$ & $50 \mathrm{~Hz}$ \\
Notch filter bandwidth $\left(B W_{N F}\right)$ & $5 \mathrm{~Hz}$ \\
\hline \hline
\end{tabular}

load-side rectifiers are disconnected and the FBS power filter works in the passive mode interacting with the current harmonics generated by the grid-side rectifier. In the mode 2 $(0.45 \mathrm{~s} \leq t<0.6 \mathrm{~s})$, the switch SW1 is closed and SW2 $=0$, which indicates that the FBS power filter works in the passive mode draining off current harmonics generated by both the grid- and load-side rectifiers. In the mode $3(t \geq$ $0.6 \mathrm{~s}$ ), the switch SW1 is closed and SW2=1, which means that the FBS power filter works in the hybrid mode interacting with current harmonics generated by both the grid- and load-side rectifiers. In all of these three operating modes, the FBS network is supplying capacitive reactive power to the grid by means of its capacitors-bank.

Figs. 7(a) and 7(b) respectively show currents in the phase $a$ and in the controlled neutral conductor of Fig. 6 for the study case A (weak grid). Ideally, when the system operates in mode 1, the FBS power filter should be just supplying conventional reactive power to the grid without filtering any current harmonics since the load rectifiers are disconnected. However, the FBS power filter interacts with the current harmonics generated by the neighbour grid-side rectifier and two interesting phenomena are observed: $i) i_{s a}$ is not perfectly sinusoidal but it is distorted by remarkable levels of $9^{\text {th }}$ - and $11^{\text {th }}$-harmonic as a consequence of a parallel resonance phenomenon occurred between the FBS power filter and the grid impedance; ii) $i_{S n}$ is not zero but it has a rms value of 0.3A since the FBS power filter offers a low impedance path for the $3^{\text {rd }}$-harmonic of the current generated by the grid-side rectifier.

The load-side rectifiers of Fig. 6 , connected at $t=0.45 \mathrm{~s}$, demands a phase current $i_{L a}$ with a rms value of $4.1 \mathrm{~A}$ and a THD about $42.2 \%$, and they inject a quite clear $3^{\text {rd }}$-harmonic current $i_{L n}$ in the neutral conductor with a rms value of 4.9A. When the system of Fig. 6 operates in mode 2 connected to a weak grid, the FBS power filter shows a rather good filtering characteristic for the $\mathrm{z}$-seq $3^{\text {rd }}$-harmonic current, albeit it works in the passive mode. Once the system reaches steady state conditions in the operating mode 2 , the rms value of $i_{S n}$ is reduced to $1,33 \mathrm{~A}$. In the operating mode 3 , the FBS power filter works in the hybrid mode and the filtering characteristic for the z-seq $3^{\text {rd }}$-harmonic current is improved even more. The rms value of $i_{S n}$ in the operating mode 3 is as low as $0.24 \mathrm{~A}$, mainly consisting of a $9^{\text {th }}$-harmonic component. Fig. 7 shows how attenuation of the $\mathrm{z}$-seq $3^{\text {rd }}$-harmonic during the operation modes 2 and 3 is not only reducing the rms value 

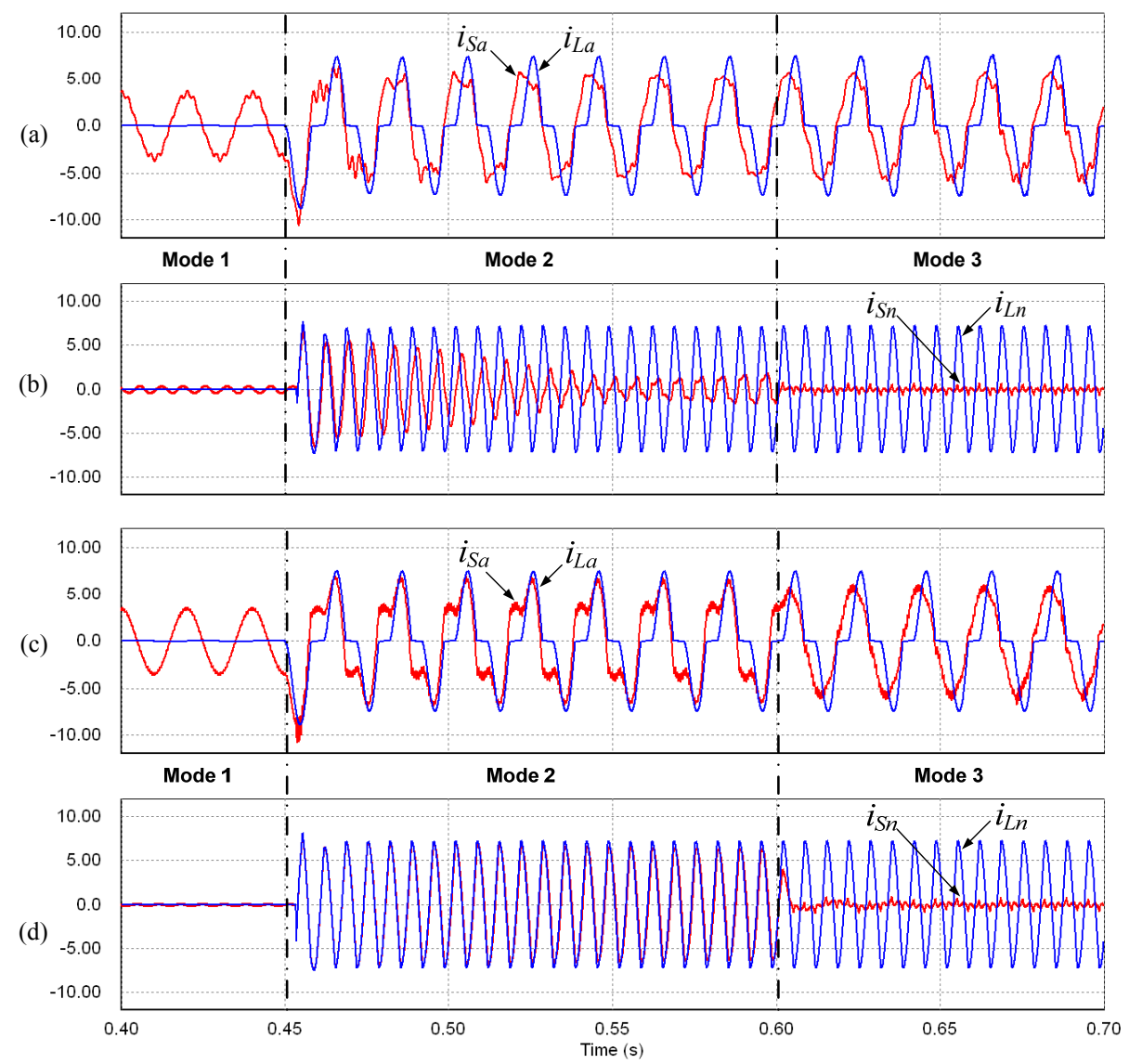

Fig. 7. Simulated results for the neutral current FBS power filter and var compensator. (a) line current in a weak grid, (b) neutral current in a weak grid, (c) line current in a strong grid, (d) neutral current in a strong grid

of the current in the controlled neutral conductor but also contributing to improve current quality in the phase conductors.

The parallel resonance phenomenon occurred between the FBS power filter and the grid impedance is excited by the pn-seq components of the current demanded by the loadside rectifiers and hence its distorting effects on the phase currents remain visible during the operation mode 3 since the single-phase VSI only improves the power filter response for z-seq current components. An analysis of the transfer function of (10) allows explaining the frequency response of FBS hybrid power filter in the three operating modes. Derived from (10), Fig. 8 describes the frequency response of the neutral current FBS power filter and var compensator in the presence of pn- and z-seq current harmonics at the load side. This figure evidences how only the $\mathrm{z}$-seq curve is modified when the power filter works in the hybrid mode since the action of the single-phase VSI only affects to the z-seq current components. It is worth to point out the high peak in the pn-seq curve at a frequency of $487 \mathrm{~Hz}$, which is responsible of the significant levels of $9^{\text {th }}$ and $11^{\text {th }}$-harmonics in the phase current at the grid-side.

Once the power filter works in the hybrid mode such resonance peak is attenuated. Fig. 8 shows the improvement in z-seq filtering capability when the power filter works in hybrid mode, which justifies the low rms value of $i_{S n}$ when the VSI is activated.

Figs. 7(c) and 7(d) show phase and neutral currents for the study case B (strong grid). In this case, the parallel resonance between the FBS power filter and the grid impedance is given at a higher frequency than in the case of a weak grid $(1.54 \mathrm{kHz})$, being its effects on $i_{s a}$ almost imperceptibles during the operating mode 1 . Therefore, $i_{S a}$ is practically sinusoidal during this period and matches to the reactive current demanded by the capacitor-bank. Moreover, $i_{S n}$ is quite low during the operation mode 1 since the low impedance of the strong grid reduces the neutral current drifted toward the FBS power filter from the grid-side rectifier. In the operation mode 2 , the FBS power filter works in the passive mode and its filtering capability is rather reduced because of the low grid impedance. The phase current $i_{S a}$ roughly results from adding the current demanded by the load-side rectifiers $i_{L a}$ to the sinusoidal reactive current demanded by capacitors-bank of the FBS filter, which gives rise to a rather distorted source current. Likewise, $i_{S n}$ is almost equal to $i_{L n}$ in steady state during the 


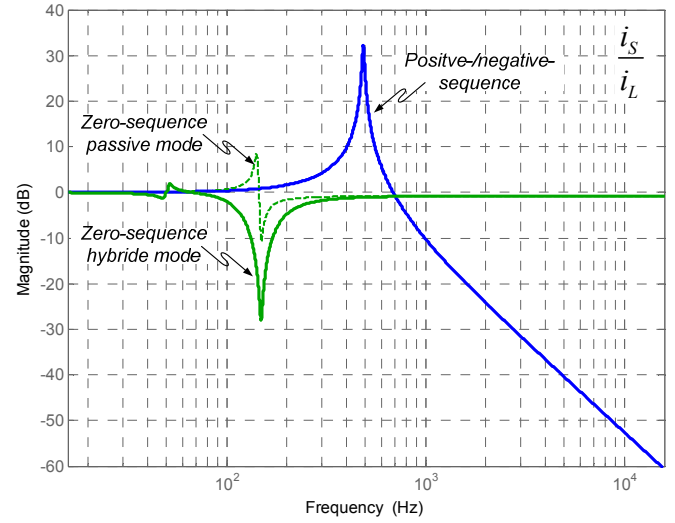

Fig. 8. Frequency response of the neutral current FBS power filter and var compensator. $\left(i_{S} / i_{L}\right)$

operating mode 2 when a strong grid is considered. Quality of the source currents is fairly enhanced when the FBS power filter works as a hybrid filter during the operating mode 3 . In this operating mode, the neutral current at the source side $i_{S n}$ is almost cancelled out and the quality of the phase currents is improved. Phase current at the grid-side presents a certain ripple during the operating modes 2 and 3 originated by the parallel resonance phenomenon between the FBS power filter and the grid impedance.

\section{EXPERIMENTAL RESULTS}

An experimental prototype was built to validate the performance of the neutral current hybrid power filter and var compensator with FBS topology of Fig. 6. MOSFET devices IRF540N 100V/33A were used to implement the single-phase VSI. This kind of switches are very suitable for the low voltage VSI of the FBS hybrid power filter because their very low on-resistance, fast switching and low-cost. The light control algorithm of Fig. 7 was programmed into a low-cost 16-bit fixed-point DSP dsPIC30F6010 running at 30MIPS. Parameters for the grid, power filter and loads in the experimental test-bed matched to those used in simulation and listed in Tables I, II and III.

A fourth operating mode was considered in the experimental evaluation of the power filter of Fig. 7. This is the operating mode 4 and results when the switch SW1 is open and SW2=1, which implies that the load-side rectifiers are disconnected and the FBS power filter works in the hybrid mode interacting with the current harmonics generated by the grid-side rectifier. The neutral current $i_{S n}$ will be almost equal zero during the operating mode 4 since the VSI of the FBS hybrid power filter is isolating the load from the grid at desired frequencies by increasing the virtual impedance in the neutral conductor linking the FBS power filter to such grid. This virtual impedance is only affecting to the neutral conductor. However, the phase currents should keep distorted even when the VSI is activated since the phase conductors are not controlled by the FBS hybrid power filter.
Fig. 9 shows some representative records of measured currents in this experimental prototype. In these scopes, a dash-dot line indicates the transition time between different operating modes. Figs. 9(a) and 9(b) respectively show currents in the phase $a$ and in the neutral conductor for the operating modes 4 and 3 when the grid was supplied by a weak $10 \mathrm{~kW}$ ac source. As expected, Fig. 9(b) evidences that neutral current $i_{S n}$ is practically equal to zero when the experimental system works in the operating mode 4 . However, the current distortion due to the parallel resonance between the capacitors-bank of the FBS power filter and the grid impedance is higher in the experimental setup than in simulation. Moreover, the experimental current $i_{S n}$ presents a small oscillation at the fundamental frequency when the system works in the operating mode 3 . It is due to the fact that load currents demanded by the three load-side rectifiers were slightly unbalanced in the experimental setup. Consequently a small current at the fundamental frequency was injected by the load rectifiers into the neutral conductor. Such current component can be appreciated in Fig. 9(b) as a small oscillation enclosing the $3^{\text {rd }}$-harmonic of the neutral current $i_{L n}$. As previously justified, such current at the fundamental frequency can not be cancelled by the FBS hybrid power filter and thus is propagated toward the source-side neutral current $i_{S n}$.

\section{CONCLUSION}

The four-branch star (FBS) topology, a new filter concept was presented in this paper. Analysis, simulations and experiments conducted in this paper proved the FBS power filter topology as an effective and economical solution for current conditioning in three-phase four-wire networks. Connection of resonant cells according to FBS topology results in independent low impedance paths for both pn- and z-seq components at specific frequencies, which allows performing selective filtering of current harmonics in both the phases and the neutral conductor of a three-phase fourwire system. The FBS power filter topology can operate in either passive or hybrid mode. In this second mode, a very simple VSI -with a dc-link voltage around $10 \%$ of the grid line voltage, extends further filtering capability of the passive network as well as it avoids overloads and unexpected resonances. A neutral current hybrid power filter and var compensator was presented in this paper as an interesting application of the FBS filtering topology for three-phase four-wire systems. The proposed solution allows low-cost implementation of the neutral current conditioning functionality in a conventional wye-connected capacitors-bank. A simple VSI and a light control algorithm were used in this work to emphasize economy and simplicity of the proposed filtering solution. Simulations together with experimental evaluation were conducted in order to evaluate the performances of the presented neutral current hybrid power filter and var compensator. Simulation 
(a)

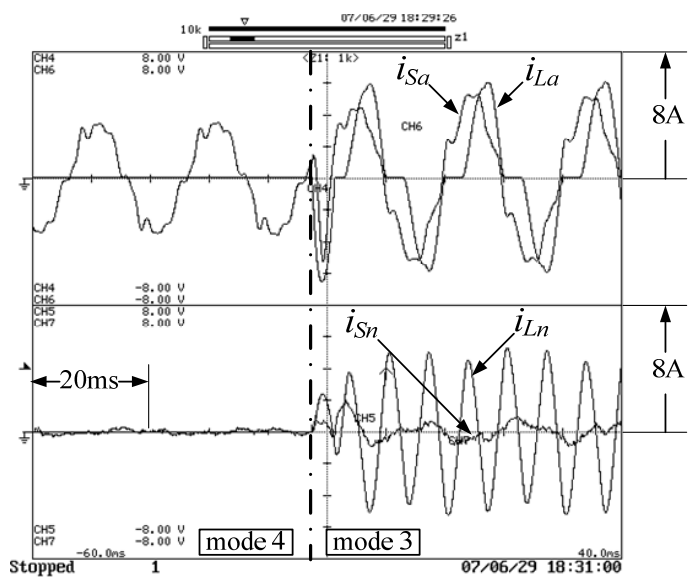

Fig. 11. Experimental source and load currents for neutral current hybri power filter and var compensator with FBS topology. (a) line current in weak grid, (b) neutral current in a weak grid.

and experimental results confirmed the good behaviour of the proposed filtering solution in cancelling out current harmonics in the neutral conductor. Performances of the proposed filtering solution can be improved by using a more complex resonant circuit with multiple resonance frequencies and/or designing independent controllers for each of the current harmonics to be filtered.

\section{ACKNOWLEDGMENT}

This work was supported by the projects ENE200806841-C02-01/ALT and ENE2008-06588-C04-03/ALT financed by the Ministerio de Ciencia e Innovacion of Spain.

\section{REFERENCES}

[1] IEEE Std. 519-1992: IEEE Recommended practices and requirements for harmonic control in electrical power systems. IEEE, 1992.

[2] Electromagnetic Compatibility (EMC), Part 3: Limits, Section 2: Limits for harmonics current emissions (equipment input current $\leq 16$ A per phase), IEC-61000-3-2, 1997.

[3] Electromagnetic Compatibility (EMC), Part 3: Limits, Section 4: Limitation of emissions of harmonics currents in low-voltage power supply systems for equipment with rated currents greater than $16 \mathrm{~A}$, IEC-61000-3-4, 1998.

[4] R. C. Dugan, M. F. McGranaghan, S. Santoso, and H. W, Beaty, Electrical Power Systems Quality, 2nd edition, New York: McGraw Hill, 2002.

[5] R. Apolonio, J.C. de Oliveira, A.B. de Vasconcellos, "Three-phase electromagnetic filter for zero sequence harmonics," in Proc. IEEE Int. Conf. Harmonic and Quality of Power, 2004, pp. 613-618.

[6] J. C. Das, "Passive filters - potentialities and limitations," IEEE Trans. Ind. App., vol. 40, pp. 232-241, Jan./Feb. 2004.

[7] F. Z. Peng and G. J. Su, "A series LC filter for harmonic compensation of ac drives," in Proc. IEEE Power Elect. Specilist Conf. (PESC'99), 1999, pp. 213-218.

[8] F. Z. Peng, "Harmonic sources and filtering approaches," IEEE Trans. Ind. App., vol.7, pp.18-25, Jul/Aug 2001.

[9] S. Choi; M. Jang, "A reduced-rating hybrid filter to suppress neutral current harmonics in three-phase four-wire systems," IEEE Trans. Ind. Electron., vol.51, pp. 927-930, Aug. 2004

[10] S. Choi, M. Jang, "Analysis and Control of a Single-Phase-InverterZigzag-Transformer Hybrid Neutral-Current Suppressor in ThreePhase Four-Wire Systems," IEEE Trans. Ind. Electron., vol.54, pp.2201-2208, Aug. 2007
[11] D. Rivas, L. Moran, J.W. Dixon, J.R. Espinoza, "Improving passive filter compensation performance with active techniques," IEEE Trans. Ind. Electron., vol.50, pp. 161-170, Feb 2003

[12] H. Fujita, T. Yamasaki, H. Akagi, "A hybrid active filter for damping of harmonic resonance in industrial power systems," IEEE Trans. Power Electron., vol.15, pp.215-222, Mar 2000

[13] M. Liserre, R. Teodorescu, F. Blaabjerg, "Multiple harmonics control for three-phase grid converter systems with the use of PI-RES current controller in a rotating frame," IEEE Trans. Power Electron., vol. 21, pp. 836-841, May 2006.

[14] D. Basic, V.S. Ramsden, P.K. Muttik, "Harmonic filtering of highpower 12-pulse rectifier loads with a selective hybrid filter system," IEEE Trans. Ind. Electron. , vol.48, pp.1118-1127, Dec 2001 